\title{
El problema del amianto en España
}

\author{
Montserrat García Gómez ${ }^{a}$
}

Entre los cancerígenos laborales, el caso del amianto es particularmente complejo. En primer lugar, algunas de las enfermedades a las que da lugar son prácticamente exclusivas del amianto: la asbestosis, las placas pleurales y el mesotelioma. En segundo lugar, el tiempo de latencia entre la exposición y la aparición de la enfermedad es extraordinariamente largo (de entre 40 y 50 años en el caso del mesotelioma). En tercer lugar, un número de casos relativamente elevado (alrededor de un 20\%) se produce en personas que no han estado expuestas profesionalmente al amianto, siendo la causa de la exposición la contaminación ambiental o la doméstica. En cuarto lugar, la forma en la que se usó y sus variadas aplicaciones han multiplicado el número de expuestos en actividades no consideradas de riesgo y/o no incluidas en el Registro de Empresas con Riesgo de Amianto (RERA). Estos casos son rechazados por el sistema compensador, que de esta manera contribuye a subestimar el impacto en la salud derivado de la exposición al amianto en nuestro país.

Carecemos de estimaciones adecuadas sobre el número de trabajadores expuestos. En 1971, las primeras estimaciones calcularon en 8.000 la cifra de expuestos en España, el $70 \%$ de ellos en el sector del fibrocemento. El Instituto Nacional de Seguridad e Higiene en el Trabajo estimó en 60.488 el número de trabajadores expuestos en 1991 y el proyecto CAREX cifró en 56.600 la cifra de expuestos a finales de dicha década. Por su parte, el Programa Integral de Vigilancia de la Salud de los Trabajadores expuestos al amianto (PIVISTEA) controlaba en diciembre de 2008 un total de 22.158 trabajadores de 14 comunidades autónomas y 306 empresas ${ }^{1}$. Entre 1977 y 2010 fallecieron en España 4.987 personas por mesotelioma, una enfermedad que en un 80-85\% de los casos en hombres tiene su origen en una exposición laboral al amianto. Actualmente mueren alrededor de 350 personas mayores de 35 años por mesotelioma al año.

La especificidad de las enfermedades derivadas de la exposición a asbesto debería facilitar su reconocimiento como profesionales. Sin embargo, desde 1962, año en que la asbes- tosis adquiere la condición de enfermedad profesional indemnizable en España, hasta el año 2010, sólo se han reconocido 815 casos $^{1}$. Somos el país europeo que menos asbestosis registra como profesional: 35 veces menos que Alemania, 21 veces menos que Bélgica, y 15 veces menos que Francia e Italia en el año $2000^{2}$. Y además, la asbestosis se encuentra actualmente en regresión en todos los países europeos excepto en España. El ascenso en el reconocimiento observado a partir de 2007 puede indicar que estamos comenzando a reflejar cifras más cercanas a la realidad. También podría indicar que los trabajos de mantenimiento, demolición y desamiantado, o cualquier otra actividad que pueda exponer a la inhalación de fibras de amianto, no están realizándose con las garantías de seguridad y salud aplicables a los mismos.

En el caso del cáncer, su reconocimiento como profesional en España es anecdótico. Hay que esperar a 1997 para encontrar el primer caso reconocido oficialmente de cáncer profesional debido al amianto. Se reconoce bajo el epígrafe "Carcinoma primitivo de bronquio o pulmón por asbestos mesotelioma pleural y mesotelioma" de la lista de enfermedades profesionales en vigor en esa fecha (la de 1978), por lo que no podemos diferenciar de cuál de los dos tipos de cáncer se trata. Desde ese año hasta el año 2011 se han notificado 164 cánceres causados por el amianto, la mitad en los dos últimos años. Si comparamos con otros países europeos, Noruega reconoce 183 veces más cáncer causado por el amianto que España y Dinamarca 133 veces más. Si nos fijamos en países más cercanos, Francia reconoce 124 veces más e Italia 41 veces más que España².

La primera consecuencia de este infra-reconocimiento es la privación a las víctimas de sus derechos en términos de prestaciones y de reconocimiento social. La segunda consecuencia es la socialización de los costes de la enfermedad, que son transferidos desde las empresas responsables de las exposiciones laborales causantes de la misma a la ciudadanía general y a los servicios de salud. La tercera consecuencia es la dificultad que genera para el desarrollo de programas de prevención de riesgos laborales eficaces.

\footnotetext{
a Subdirección General de Sanidad Ambiental y Salud Laboral. Ministerio de Sanidad, Servicios Sociales e Igualdad. España.
}

\author{
Correspondencia: \\ Montserrat García Gómez. \\ Ministerio de Sanidad, Servicios Sociales e Igualdad. \\ Paseo del Prado 18-20 \\ 028014 Madrid \\ mgarciag@msssi.es
}


A los límites de la legislación sobre enfermedades profesionales en materia de indemnizaciones, así como a la rigidez de sus procedimientos, se añade un problema adicional: el carácter no laboral de muchas de las enfermedades producidas, pues parte de las víctimas no han contraído la enfermedad a través de una relación laboral, sino que su afectación se ha producido bien por la contaminación ambiental provocada por la proximidad de una instalación contaminante, bien por el contacto doméstico con el amianto por las ropas contaminadas de algún pariente que trabajaba en una de dichas instalaciones. En ese caso los sistemas de compensación vigentes son absolutamente inoperantes y las víctimas tienen la litigación por daños y perjuicios contra la empresa como único camino para el resarcimiento. La principal medida implementada en otros países para paliar estas carencias del sistema compensador ha sido la creación de fondos de compensación sin culpa específicos para las víctimas el amianto ${ }^{3}$.

Una de las principales dificultades para la puesta en marcha del Programa Integral de Vigilancia de la Salud de los Trabajadores Expuestos al Amianto (PIVISTEA) fue, y sigue siendo, la elaboración del registro de trabajadores que habían estado expuestos a asbesto.

Vicent Villanueva y colaboradores ${ }^{4}$ publican en este número de Archivos una evaluación del grado de cumplimiento de la ficha de vigilancia de la salud de los trabajadores expuestos a amianto, y ponen las bases para un análisis muy poco frecuente en España y que es absolutamente necesario. Sobre una muestra de 400 fichas cumplimentadas por los servicios de prevención, evalúan la exhaustividad de la cumplimentación de los datos de la ficha del anexo $\mathrm{V}$ del Real Decreto 396/2006, concluyendo que, lamentablemente, es insuficiente en la actualidad.

Cabe recordar que la forma en la que se usó el amianto y sus aplicaciones plantean el problema de los trabajos con riesgo de exposición a amianto en la actualidad. Estos trabajos comprenden fundamentalmente la retirada del amianto instalado, los trabajos de mantenimiento y reparación sobre materiales que contienen amianto, y las actividades de transporte, tratamiento y/o destrucción de residuos que contienen asbesto, procedente del amianto instalado en infraestructuras urbanas de toda índole.
Recientemente el Parlamento Europeo ha aprobado una Propuesta de Resolución sobre los riesgos para la salud en el lugar de trabajo relacionados con el amianto y sobre las perspectivas de eliminación de todo el amianto existente (2012/2065(INI)), destacando los problemas para la detección y registro del amianto instalado; la cualificación y formación de los profesionales, empresarios y trabajadores; el desarrollo de programas de eliminación; los criterios para el reconocimiento de las enfermedades relacionadas con el amianto; el apoyo para grupos de víctimas del amianto; y las estrategias para la prohibición mundial del amianto.

Villanueva y colaboradores nos recuerdan en su artículo ${ }^{4}$ que el amianto constituye un problema de salud pública relevante, cuyo abordaje incluye acciones como la vigilancia de los colectivos expuestos en la actualidad y en el pasado y la indemnización de las víctimas.

El análisis de la cumplimentación de las fichas oficiales de vigilancia de la salud de los trabajadores del amianto forma parte de los cometidos de la administración sanitaria, dirigidos, entre otros objetivos, al control de la actividad sanitaria de prevención de riesgos laborales para mejorar su efectividad y asegurar el adecuado tratamiento de las alteraciones de la salud detectadas y su reconocimiento médicolegal. Las enormes diferencias entre los Estados miembros de la Unión Europea para el reconocimiento de las enfermedades profesionales derivadas de la exposición al amianto son inaceptables. Es absolutamente imprescindible mejorar dicha cumplimentación si queremos alcanzar esos objetivos.

\section{BIBLIOGRAFÍA}

1. García Gómez M, Menéndez Navarro A, Castañeda López R. Incidencia en España de la asbestosis y otras enfermedades pulmonares benignas debidas al amianto durante el período 1962-2010. Rev Esp Salud Pública. 2012;86:613-25.

2. Eurogip. Les maladies profesionnelles liées à l'amiante en Europe. Reconnaissance - Chiffres - Dispositifs spécifiques. Paris: Eurogip; 2006.

3. Azagra Malo A. La tragedia del amianto y el derecho español. Barcelona: Atelier; 2007.

3. Villanueva Ballester V, Damiá Iborra M, Esteban Buedo V. Exhaustividad de la cumplimentación de la ficha de vigilancia de la salud de los trabajadores expuestos a amianto. Arch Prev Riesgos Labor. 2013;16:77-81. 